\section{CPC-032 CLOPIDOGREL FOR THE TREATMENT OF CHILDREN WITH A SYSTEMIC-TO-PULMONARY ARTERIAL SHUNT}

doi:10.1136/ejhpharm-2013-000276.489

${ }^{1}$ A Garzone, 'F Ciuccarelli, 'A Pompilio, 'E Andresciani, ${ }^{2} F$ Pinto, ${ }^{2} \mathrm{MS}$ De Meo, ${ }^{1} \mathrm{MC}$ Cerlesi, ${ }^{3} \mathrm{P}$ Colonna, ${ }^{3} \mathrm{E}$ Franchi, 'V Moretti, 'L Carloni, ${ }^{1} \mathrm{P}$ Marzioni, 'M Buccolini. ${ }^{1}$ AO Ospedali Riuniti - Presidio G. Salesi, Farmacia, Ancona, Italy; ${ }^{2}$ Università degli Studi di Camerino, Scuola di Specializzazione in Farmacia Ospedaliera, Camerino, Italy; ${ }^{3}$ AO Ospedali Riuniti - Presidio G.M. Lancisi, Cardiochirurgia e Cardiologia Pediatrica e Congenita, Ancona, Italy

Background Clopidogrel is a thienopyridine drug that acts by binding selectively and irreversibly to the adenosine diphosphate P2Y12 receptor on platelets. Platelet aggregation is consequently inhibited. Clopidogrel is used to prevent ischemic events in patients at risk, when other drugs fail or are not tolerated. Paediatric use is not authorised because of limited information about efficacy and safety.

Purpose To illustrate our experience in the three-month use of clopidogrel in children with a systemic-to-pulmonary arterial shunt, prior to definitive surgical intervention.

Materials and Methods On February 2012 a temporary systemicto-pulmonary arterial shunt was placed in a four-month-old patient, affected by Tetralogy of Fallot with hypoplasia of the infundibulum and pulmonary valve. We administered two antiaggregant drugs, ASA $18 \mathrm{mg}$ and clopidogrel $0.2 \mathrm{mg} / \mathrm{Kg}$ once a day, because of the high risk of thrombotic closure of the shunt. We chose an extemporaneous preparation of type 5 capsules with lactose as a diluent. After that, four other children (from 2 months to 4 years old) were treated with clopidogrel, mostly in association with ASA or together with enoxaparin.

Results We prepared capsules from 0.75 to $3.5 \mathrm{mg}$. For all patients we obtained authorization by the Ethics Committee and the parent's informed consent. After surgery, the children were observed for 7-8 days depending on clinical follow up and complications. We checked the blood count and shunt patency with clinical observation, analysis and echocardiogram. After discharge, patients were first recalled 10-15 days later, then after 1-2 months to see the doctor, have an ECG, blood tests and echocardiogram. Pt was not necessary. No serious side effects were observed.

Conclusions Paediatric clopidogrel treatment is rapidly increasing. A wider number of cases, a comparison with other professional experience, and, most of all, controlled clinical trials, would be desirable.

No conflict of interest.

\section{CPC-033 COMPARING EFFECTIVENESS OF FIRST-LINE DISEASE MODIFYING DRUGS IN RELAPSING-REMITTING MULTIPLE SCLEROSIS PATIENTS}

doi:10.1136/ejhpharm-2013-000276.490

H Esteban-Cartelle, E Espino-Paisan, C Crespo-Diz, T Rodríguez-Jato, P Suarez Artime. Complejo Hospitalario Universitario de Santiago de Compostela, Pharmacy, Santiago de Compostela, Spain

Background Immunomodulatory drugs represent the best therapeutic option in first-line treatment of relapsing-remitting multiple sclerosis (RRMS). This group includes glatiramer acetate (GA) and three formulations of Interferon $\beta$ (IFN $\beta$ ): subcutaneous IFN $\beta 1$ a (scIFN $\beta 1 a)$, intramuscular IFN $\beta 1$ a (imIFN $\beta 1 a)$ and subcutaneous IFN $\beta 1 b$ (scIFN $\beta 1 b)$. Several studies have reported similar efficacy among IFN $\beta$ and GA preparations, while other have concluded that some differences exist between them.

Purpose To compare the effectiveness of first-line disease modifying drugs (FL-DMD) in patients with RRMS.
Materials and Methods In this retrospective and observational study we included treatment-naive patients with RRMS who had started treatment with FL-DMD between 1996 and 2011. Patients receiving other immunosuppressant drugs were excluded. Data were collected from medical records and pharmacy computer applications. Patients were classified in four groups: those treated with imIFN $\beta 1 \mathrm{a}$, scIFN $\beta 1 \mathrm{a}$, scIFN $\beta 1 \mathrm{~b}$ or GA. The annualised relapse rate (ARR) and degree of disability for each group were determined in the pre-treatment and treatment periods. The time to first relapse (TFR), proportion of relapse-free patients (RFP) and proportion of patients without progression of disability (DPFP) in each group were compared.

Results We identified 72 patients who had started treatment with DMD: 22 with imIFN $\beta 1$ a, 13 with scIFN $\beta 1$ a, 26 with scIFN $\beta 1 b$ and 11 with AG.

Mean ARR in the pre-treatment and treatment periods were the following (mean \pm SD): $0.73 \pm 0.40$ and $0.10 \pm 0.16$ for imIFN $\beta 1 \mathrm{a}$. $0.81 \pm 0.43$ and $0.21 \pm 0.32$ for scIFN $\beta 1$ a. $0.56 \pm 0.33$ and $0.11 \pm 0.20$ for scIFN $\beta 1 b$ and $0.73 \pm 0.34$ and $0.17 \pm 0.28$ for GA. TFR in each group were respectively $5.8 \pm 4.2$. $3.8 \pm 2.8 .5 .3 \pm 3.8$ and $2.4 \pm 1.6$ years. We found $59.1 \%, 61.5 \%, 61.5 \%$ and $63.6 \%$ RFP in each group and $90.9 \%, 92.3 \%, 88.5 \%$ and $81.8 \%$ DPFP respectively. No statistical differences were found in TFR, RFP or DPFP among groups.

Conclusions No differences were found among the different firstline treatment options in terms of reduction of disease activity or progression of disability.

No conflict of interest.

\section{CPC-034 COMPARISON OF EFFECTIVENESS AND SAFETY OF PRIMARY PROPHYLAXIS WITH BIOSIMILAR FILGRASTIM VS REFERENCE MEDICINAL PRODUCT IN PATIENTS TREATED WITH R-CHOP}

doi:10.1136/ejhpharm-2013-000276.491

M Isidoro, V Domingos, A Melo Gouveia. Instituto Português Oncologia Lisboa, Pharmacy, Lisbon, Portugal

Background Prophylactic administration of filgrastim can reduce the risk of febrile neutropenia (FN) in oncological patients undergoing myelosuppressive chemotherapy.

According to approved guidance on biosimilar drugs, clinical comparability can be obtained from results extrapolated from other indications.

Due to this simplified pathway, concerns about safety and effectiveness in some clinical settings may remain.

Purpose To compare the effectiveness and safety of biosimilar filgrastim (B) with the reference medicinal product (RMP) in patients with haematological malignancies treated with the $\mathrm{R}-\mathrm{CHOP}$ regimen and undergoing primary prophylaxis (PP) with filgrastim.

Materials and Methods A single-centre, retrospective and transversal study was performed: from March 2010 to October 2010 (RMP) and March 2011 to February 2012 (B).

Main endpoints were incidence of FN (per patient and per cycle), time to absolute neutrophil count (ANC) recovery, incidence of NP in first cycle (1stCy), number of hospitalisation days related to FN Other endpoints and patient-related risk factors for FN were evaluated.

Results The results seem to support equivalence of effectiveness between these drugs. We did not find any safety-related incidents. More patients are being included to increase the sample size and allow statistical significance. These results may further support the use of the more cost-efficient option.

No conflict of interest. 